\title{
On the model of Teflon ablation in an ablation-controlled discharge
}

\author{
Michael Keidar ${ }^{1}$, Iain D Boyd ${ }^{1}$ and Isak I Beilis ${ }^{2}$ \\ ${ }^{1}$ Department of Aerospace Engineering University of Michigan, Ann Arbor, MI 48109, USA \\ ${ }^{2}$ Electrical Discharge and Plasma Laboratory, Fleischman Faculty of Engineering, \\ Tel Aviv University, POB 39040, Tel Aviv 69978, Israel
}

Received 19 March 2001

\begin{abstract}
A kinetic model is developed of Teflon ablation caused by a plasma. The model takes into account the returned atom flux that forms in the non-equilibrium layer during the ablation. This approach makes it possible to calculate the ablation rate for the case when the Teflon surface temperature and the density and temperature in the plasma bulk are known.
\end{abstract}

The problem of the ablation controlled discharge has a common general interest in a number of applications such as electric fuses, circuit breakers, soft x-ray, pulsed plasma thrusters and extreme ultraviolet sources [1-5]. In these devices, the discharge energy is principally dissipated by ablation of wall material, which then forms the main component of the discharge plasma. The ablated vapour increases the pressure within the capillary and the plasma is expelled through the exit.

Previously, most of the plasma models of the ablated controlled discharge employed the Langmuir relationship [6], which is limited to the case of material ablation into a vacuum. For the conditions of a pulsed plasma thruster (PPT) this approach was also recently used [7,8]. However, the process of ablation in the ablation controlled discharge should be described taking into account the fact that in the Teflon cavity the vapour does not expand into vacuum but rather into a volume discharge. For application to the vacuum arc discharge, a kinetic model of the cathode vapourization in the nonequilibrium plasma layer was developed by Beilis $[9,10]$. It was shown that the flux of returned atoms toward the surface can become comparable to the flux of vaporizing atoms. It was also concluded $[9,10]$ that the parameters at the outer boundary of the kinetic layer are close to their equilibrium values and that the velocity at the outer boundary of the kinetic layer is much smaller than the sound velocity. Therefore, it was found that the cathode erosion rate in an arc discharge by vapourization is much smaller than the solid body evaporation rate into vacuum. In the present work we employ a kinetic approach similar to that used for the cathode vacuum arc evaporation to calculate Teflon ablation parameters. As an example, the conditions typical for an electrothermal PPT are considered [4, 7].

The problem starts by considering the non-equilibrium kinetic layer near the evaporating surface. Let us consider the structure of the near surface layers in detail as shown in figure 1. One can distinguish two different layers between

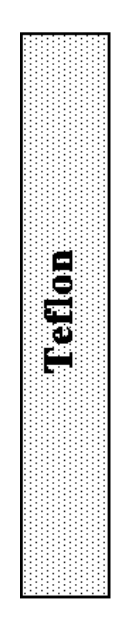

(1)

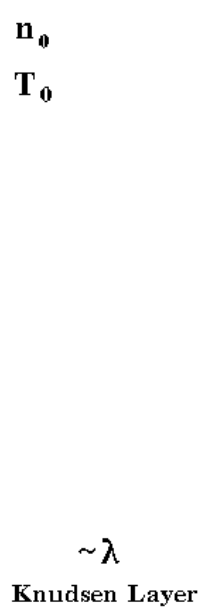

(1)

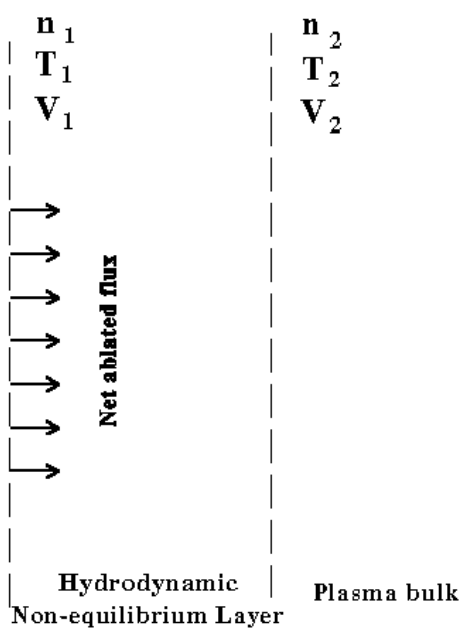

(1)

(2)
Figure 1. Schematic representation of the layer structure near the ablated surface.

the surface and the plasma bulk: (1) a kinetic non-equilibrium layer adjusted to the surface with a thickness of a few mean free paths (the Knudsen layer), (2) a collision-dominated non-equilibrium layer, where the electron and heavy particle temperatures differ. It is assumed that at the right edge of the second layer, all species (ions, neutrals and electrons) reach thermal equilibrium. The basic idea of the present model consists in combining the model for the kinetic layer [9-11] with hydrodynamic layer analyses in the second layer. Use of the general plasma model [7] in the equilibrium region provides the electron temperature $T_{2}$ and plasma density $n_{2}$. Firstly, we briefly introduce the kinetic model of the nonequilibrium layer. According to the approach of the work in [9] and [10] (using Anisimov's [11] assumption that the velocity distribution function for the returned particles is 
$\beta f_{1}(\boldsymbol{V})$, where $\beta$ is a proportionality coefficient and $f_{1}(\boldsymbol{V})$ is the Maxwellian distribution function shifted by $V_{1}, V$ is the velocity vector) the relation of the heavy particle parameters at the outer boundary of the kinetic layer in the case of arbitrary velocity $V_{1}$ are as described by the following set of equations:

$$
\begin{aligned}
& \frac{n_{0}}{2\left(\pi d_{0}\right)^{0.5}}=n_{1} V_{1}+\beta \frac{n_{1}}{2\left(\pi d_{1}\right)^{0.5}}\left\{\exp \left(-\alpha^{2}\right)-\alpha \pi^{0.5} \operatorname{erfc}(\alpha)\right\} \\
& \frac{n_{0}}{4 d_{0}}=\frac{n_{1}}{2 d_{1}}\left\{\left(1+2 \alpha^{2}\right)-\beta\left[\left(0.5+\alpha^{2}\right) \operatorname{erfc}(\alpha)\right.\right. \\
& \left.\quad-\alpha \exp \left(-\alpha^{2}\right) / \pi^{0.5}\right\} \\
& \frac{n_{0}}{\left(\pi d_{0}\right)^{1.5}}=\frac{n_{1}}{\left(d_{1}\right)^{1.5}} \pi^{-1}\left[\alpha\left(\alpha^{2}+2.5\right)\right. \\
& \quad-(\beta / 2)\left\{\left(2.5+\alpha^{2}\right) \alpha \operatorname{erfc}(\alpha)-\left(2+\alpha^{2}\right) \exp \left(-\alpha^{2}\right) / \pi^{0.5}\right]
\end{aligned}
$$

where $d_{0}=m / 2 k T_{0}, d_{1}=m / 2 k T_{1}, \alpha=V_{1} /\left(2 k T_{1} / m\right)^{0.5}$, $\operatorname{erfc}(\alpha)=1-\operatorname{erf}(\alpha), \operatorname{erf}(\alpha)$ is the error function, $T_{0}$ is the surface temperature and $n_{0}$ is the equilibrium density.

Very recently it was shown that the velocity at the outer boundary of the kinetic layer $V_{1}$ strongly affects the kinetic layer parameters [12]. To find the velocity $V_{1}$ we apply the mass and momentum conservation equations for heavy particles in the hydrodynamic region (assuming a single fluid model) between boundaries 2 and 3 . Assuming weakly ionized plasma in the hydrodynamic layer, the integration of the mass and momentum conservation equations yields the following relations between parameters at boundaries 2 and 3:

$$
\begin{aligned}
n_{1} V_{1} & =n_{2} V_{2} \\
n_{1} k T_{1}+m n_{1} V_{1}^{2} & =n_{2} k T_{2}+m n_{2} V_{2}^{2} .
\end{aligned}
$$

Combination of these two equations yields the following expression for the velocity at the outer boundary of the kinetic (Knudsen) layer:

$$
V_{1}^{2} /\left(2 k T_{1} / m\right)=\left(T_{2} n_{2} / 2 T_{1}-n_{1} / 2\right) /\left(n_{1}-n_{1}^{2} / n_{2}\right) .
$$

This equation makes it possible to calculate the velocity at boundary 1 and therefore to calculate the ablation rate that is proportional to $V_{1} n_{1}$. The system of equations is closed if the equilibrium vapour pressure can be specified. In the case of Teflon, the equilibrium pressure formula is used:

$$
P=P_{c} \exp \left(-T_{c} / T_{0}\right)
$$

where $P=n_{0} k T_{0}$ is the equilibrium pressure, $P_{c}=1.84 \times$ $10^{20} \mathrm{~N} \mathrm{~m}^{-2}$ and $T_{c}=20815 \mathrm{~K}$ are the characteristic pressure and temperature, respectively [4].

The solution of the problem depends upon plasma density $n_{2}$, plasma temperature $T_{2}$ and surface temperature $T_{0}$. The parameters $n_{2}, T_{2}$ are determined by the bulk plasma flow. It was estimated from various experiments that, under typical PPT operation conditions, the plasma density near the Teflon surface is about $10^{21}-10^{24} \mathrm{~m}^{-3}$, the plasma temperature is about $1-4 \mathrm{eV}$ and the Teflon surface temperature $T_{0}$ is about $600-650 \mathrm{~K}[4,7,8,13]$. In the present paper we present solutions with $n_{2}, T_{2}$ and $T_{0}$ as parameters in the ranges mentioned above.

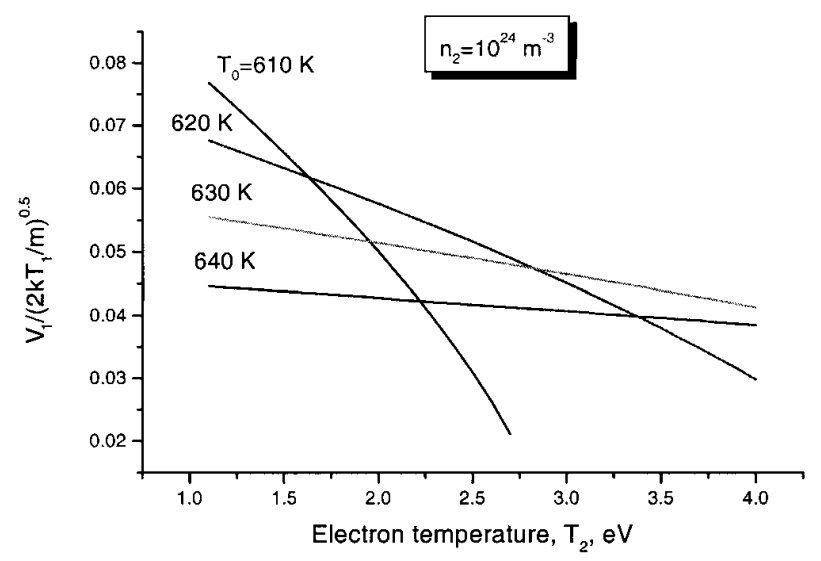

Figure 2. The velocity $V_{1}$ as a function of plasma temperature with Teflon surface temperature as a parameter.

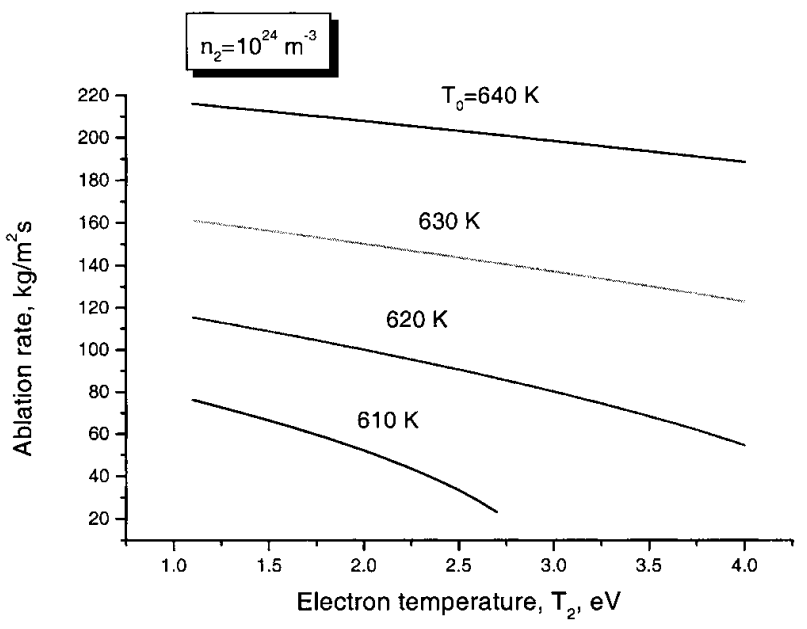

Figure 3. The ablation rate as a function of plasma temperature with Teflon surface temperature as a parameter.

The dependence of the velocity $V_{1}$ on the electron temperature $T_{2}$ is shown in figure 2 with surface temperature $T_{0}$ as a parameter for given $n_{2}$. One can see that the velocity $V_{1}$ remains small over the entire range of plasma temperature and generally decreases with temperature increase. The velocity $V_{1}$ is very sensitive to the plasma temperature variation in the case of relatively small surface temperature. As a result of this dependence, the ablation rate also decreases with increasing electron temperature as shown in figure 3.

Ablation rate contours in the plane with the plasma density and Teflon surface temperature as the coordinates are displayed in figure 4. The same ablation rate that can be found in the high and low density range corresponds to the solution of the problem with small and large velocity at the outer boundary of the kinetic layer, respectively. There is no solution for the ablation rate in regions above the curve with ablation rate equal to zero. This region in the $n_{2}-T_{0}$ plane corresponds to the case when the right-hand side in equation (4) is negative. The physical meaning of these results can be explained as follows. In the ablation-controlled discharge, the plasma density in the bulk is determined by the rate of ablation from the surface. In the case of small surface temperature one can expect a smaller ablation rate and therefore high plasma densities in the discharge cannot be generated. 


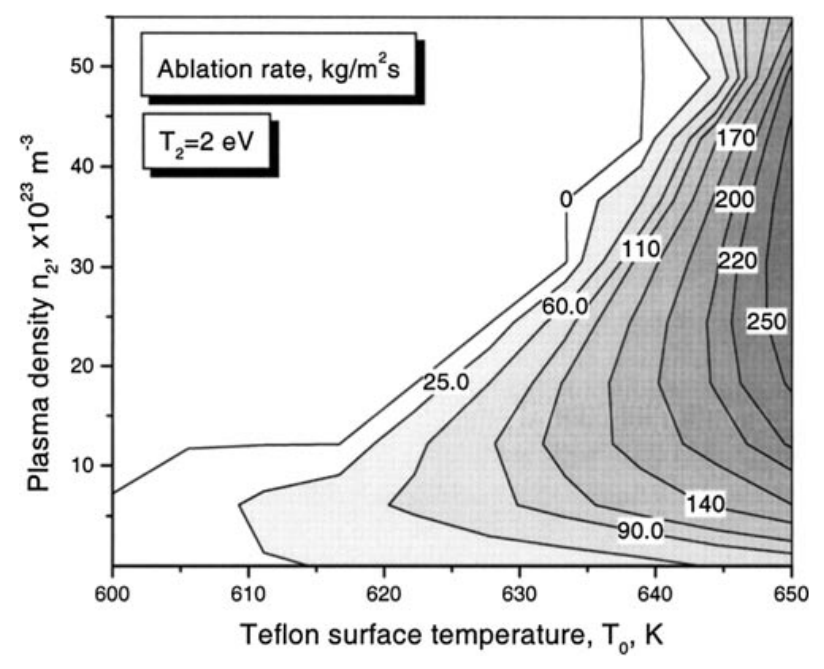

Figure 4. Contours of ablation rate in the plasma density $\left(n_{2}\right)$-Teflon surface temperature $\left(T_{0}\right)$ plane.

It is important to note that the present model predicts the dependence of the ablation rate on the plasma bulk density, electron temperature and the surface temperature. It is also found that the flow velocity at the outer boundary of the kinetic layer (that determines the ablation rate) is smaller than the sound velocity under typical PPT conditions. In this sense the present model is different from previously used approaches $[7,8]$, where the ablation rate was determined by the surface temperature only and the sonic velocity was assumed at the outer boundary of the kinetic layer.

In the typical range of PPT parameters considered the maximum ablation rate was calculated to be about $300 \mathrm{~kg} \mathrm{~m}^{-2} \mathrm{~s}^{-1}$. It should be noted that the average ablation rate measured in experiments is in the range $1-40 \mathrm{~kg} \mathrm{~m}^{-2} \mathrm{~s}^{-1}$ depending on the parameters $n_{2}, T_{2}$ for different thruster types $[4,13]$. During the discharge pulse, the plasma parameters vary so that near the current peak one can expect an ablation rate higher than average while towards the end of the pulse the ablation rate decreases. The time-averaged ablated mass calculated using this model for baseline PPT- 4 is about
$24 \mathrm{~kg} \mathrm{~m}^{-2} \mathrm{~s}^{-1}$, which is close to that measured in the experiment ( $\left.\sim 29 \mathrm{~kg} \mathrm{~m}^{-2} \mathrm{~s}^{-1},[13]\right)$.

In summary, we have developed a kinetic model of the material ablation in an ablated controlled discharge with application to a pulsed plasma thruster. The model accounts for the case when the velocity at the outer boundary of the kinetic layer is smaller than the sound velocity due to the presence of a high-density discharge plasma. The present model can be coupled with a plasma discharge model to describe the electrical discharge self-consistently.

\section{Acknowledgments}

The first two authors gratefully acknowledge the financial support of the Air Force Office of Scientific Research through grant F49620-99-1-0040.

\section{References}

[1] Muller L 1993 J. Phys. D: Appl. Phys. 261253

[2] Domejean E, Chevrier P, Fievet C and Petit P 1997 J. Phys. D: Appl. Phys. 302132

[3] Kukhlevsky S V, Kaiser J, Samek O, Liska M and Erostyak J 2000 J. Phys. D: Appl. Phys. 331090

[4] Burton R L and Turchi P J 1998 J. Prop. Power 14716

[5] Hong D, Dussart R, Cachoncinlle C, Rosenfeld W, Gotze S, Pons J, Viladrosa R, Fleurier C and Pouvesle J M 2000 Rev. Sci. Instrum. 7115

[6] Langmuir I 1913 Phys. Rev. 2329

[7] Keidar M, Boyd I D and Beilis I I 2000 IEEE Trans. Plasma Sci. 27376

[8] Mikellides Y G 1999 Theoretical modeling and optimization of ablation-fed pulsed plasma thruster PhD Thesis The Ohio State University

[9] Beilis I I 1985 IEEE Trans. Plasma Sci. 13288

[10] Beilis I I 1995 Theoretical modeling of cathode spot phenomena Vacuum Arc Science and Technology ed R L Boxman, P Martin and D Sanders (Park Ridge, NJ: Noyes)

[11] Anisimov S I 1968 Sov. Phys.-JETP 27182

[12] Keidar M, Fan J, Boyd I D and Beilis I I 2001 J. Appl. Phys. 89 3095

[13] Burton R L and Bushman S S 1999 AIAA Paper 99-2288 